\title{
Cuidados de enfermería en pacientes crónicos en el área de emergencia: una revisión sistemática
}

\author{
María Fernanda Navas Valdez \\ fernanditamaria10@hotmail.com \\ https://orcid.org/0000-0003-1908-8017 \\ Universidad Técnica de Ambato \\ Ambato-Ecuador \\ Herrera López José Luis ${ }^{2}$ \\ Docente de la Carrera de Enfermería \\ J1.herrera@uta.edu.ec \\ http://orcid.org/0000-0002-6061-0156 \\ Universidad Técnica de Ambato, Ecuador
}

\section{RESUMEN}

Introducción: La atención de pacientes por enfermedades crónicas es más prevalente por el incremento de población adulta mayor, de mano con el cambio de hábitos de consumo y actividad que potencia la aparición de estas enfermedades. En este sentido, resulta de interés conocer los protocolos y procedimientos recomendados en el área de emergencia para este tipo de enfermedades.

Objetivo: Describir los cuidados de enfermería en pacientes crónicos que son atendidos en el área de emergencia, mediante una revisión sistémica de la bibliografía.

Método: Revisión sistémica de la literatura mediante la selección de artículo académicos con el método PRISMA.

Resultados: Se seleccionaron 14 publicaciones acomodadas a las palabras clave de búsqueda y criterios de inclusión y exclusión considerados para su selección.

Conclusiones: La existencia de protocolos y métodos garantiza el adecuado funcionamiento en las unidades de emergencia. Se requiere la priorización de diagnósticos para su atención, los cuales están generalmente relacionados con complicaciones de la propia enfermedad crónica que pueden ser prevenidos. El enfermero deberá en algunos casos priorizar la atención de los diagnósticos más emergentes de acuerdo a la condición del paciente.

Palabras clave: cuidados de enfermería; paciente crónico; emergencia; revisión sistémica. 


\title{
Nursing care in chronic patients in the emergency area: a systematic review
}

\begin{abstract}
Introduction: The care of patients for chronic diseases is more prevalent due to the increase in the older adult population, hand in hand with the change in consumption habits and activity that enhances the appearance of these diseases. In this sense, it is of interest to know the protocols and recommended procedures in the emergency area for this type of disease.
\end{abstract}

Objective: To describe nursing care in chronic patients who are cared for in the emergency area, through a systemic review of the bibliography.

Method: Systemic review of the literature by selecting academic articles with the PRISMA method.

Results: 14 publications were selected according to the search keywords and inclusion and exclusion criteria considered for their selection.

Conclusions: The existence of protocols and methods guarantees the adequate operation in the emergency units. Prioritization of diagnoses is required for their care, which are generally related to complications of the chronic disease itself that can be prevented. In some cases, the nurse must prioritize the attention of the most emerging diagnoses according to the patient's condition.

Key words: nursing care; chronic patient; emergency; systemic review.

Artículo recibido: 05 octubre. 2021 Aceptado para publicación: 02 noviembre 2021 Correspondencia: fernanditamaria10@hotmail.com Conflictos de Interés: Ninguna que declarar 


\section{INTRODUCCIÓN}

Los cuidados y atención de enfermería están sujetos al área en el que son aplicados.

Particularmente, el área de emergencia representa un espacio de atención de enfermería en el cual se requieren protocolos establecidos que permitan el accionar inmediato, ya que el manejo del tiempo en situaciones de emergencia es uno de los factores más importantes. Las enfermedades crónicas son en la actualidad entre las que más generan atención de emergencia, sobre todo en los grupos de adultos mayores, en los que este tipo de enfermedades son más frecuentes.

Los sistemas de salud en la actualidad enfrentan una problemática que deriva del cambio en la distribución demográfica que ha venido dándose en las últimas décadas, caracterizado por un incremento de la población de adultos mayores, grupo poblacional que actualmente representa aproximadamente el 13,1\% de la población en el continente americano, y que se estima que para el año 2025 alcance un incremento del 18,6\% (1). Las estimaciones a nivel mundial indican que el porcentaje de adultos mayores alcanzará aproximadamente un $20 \%$.

Este cambio en la composición demográfica significa un desafío para la salud pública, debido a que las exigencias en cuanto a salud son diferentes de acuerdo a las edades, por lo que es razonable pensar que un niño y un adulto mayor, requieren diferentes tipos de atención y estarán caracterizados por diferentes perfiles epidemiológicos. Ahora bien, el envejecimiento trae consigo la aparición de enfermedades de todo tipo, entre ellas, enfermedades crónicas que requieren mayor demanda de la atención médica y de enfermería.

La aparición de enfermedades crónicas no solamente está adjudicada al incremento en la edad que alcanzan a vivir las personas, sino que también se relaciona estrechamente en los cambios de hábitos de vida enfocados sobre todo en la alimentación, actividad física y consumo de diferentes sustancias (alcohol, cigarrillo, entre otras) que pueden afectar la salud del individuo. En este sentido, se observa en la actualidad la convergencia de estos factores: hábitos de vida poco saludables, incremento en el promedio de vida; lo cual da como resultado el incremento de enfermedades de carácter crónico, que demandan atención del personal médico (2).

Las enfermedades crónicas se definen como enfermedades de larga duración y progresivas e incurables. Generalmente, estas enfermedades se presentan como cardíacas, 
cancerígenas, respiratorias, y diabetes, siendo estos elementos los más prevalentes, así como constituyen los factores de mortalidad más prevalentes a nivel mundial (3)-

La pandemia por Covid-19 que caracterizó al año 2020, y genero impacto a nivel mundial, hizo más evidente aún los grados de susceptibilidad que generan las enfermedades crónicas en adultos mayores, ya que en estos casos se alcanzaba el mayor índice de mortalidad, presentándose manifestaciones atípicas del virus que en muchos casos llevó a la muerte de los pacientes (4).

Desde la formación del área de enfermería, resulta relevante comprender las acciones en la atención de enfermedades, ya que estas patologías, de acuerdo a sus características presentan exigencias específicas, relacionadas con la dieta, atención farmacológica, apoyo psico-social, entre otros.

Las investigaciones en torno a las enfermedades crónicas afirman que muchas enfermedades de este tipo generan grados de dependencia que se incrementan con la edad, lo que hace que los servicios y atención brindados exijan más tiempo, recursos, medicamentos, etc. Además, se observa que existe alto grado de vinculación de la aparición de estas enfermedades y afecciones en la vivencia emocional que en grados más graves puede alcanzar trastornos depresivos y relacionados con el estado de ánimo e inclusive, en algunos casos, que afectan también los procesos cognitivos (3).

En base a este antecedente, se comprende que las enfermedades crónicas son complejas, de alto riesgo y requieren un alto uso de recursos técnicos, tecnológicos, de personal, económicos. Además, están en incremento ya que se asocian con la edad de las personas, y se presencia un incremento en la población adulta mayor a nivel mundial, y que generan grados de dependencia y pueden asociarse a enfermedades relacionadas con el estado emocional, cognitivo, que pueden alcanzar grados depresivos.

La convergencia de estos factores hacen que los cuidados brindado para este tipo de enfermedades sean más específicos; considerando que es un grupo poblacional en incremento, existen muchos factores que se van sumando a la teoría desarrollada en la rama de la enfermería, sobre la atención a este tipo de enfermedades, por lo que resulta necesario estudiar el estado del arte en cuanto al cuidado de enfermería en enfermedades crónicas, con objeto de comprender los avances que se han dado, las exigencias todavía existentes y las proyecciones en cuanto a este tipo de atención en salud. 
Consecuentemente, la presente investigación tiene como objetivo describir los cuidados de enfermería en pacientes crónicos que son atendidos en el área de emergencia, mediante una revisión sistémica de la bibliografía.

\section{ESTRATEGIAS METODOLÓGICAS O MATERIALES Y MÉTODOS}

En los registros y protocolos de la ciudad de Ambato, no existen investigaciones referentes a la temática propuesta, en la metodología de investigación sistemática.

La metodología de investigación propuesta en esta investigación fue la de la Revisión Sistemática (R.S.). El método que se utilizó para la recolección y clasificación de información, fue el recomendado método PRISMA, para garantizar la rigurosidad y reproducción de la investigación en otros contextos.

Los criterios aplicados para la selección de artículos fueron los que se describen a continuación:

\section{Se incluyeron los artículos que:}

- Concuerdan con los criterios de elegibilidad basados en las palabras calve: "atención en enfermería" "emergencia" "enfermedades crónicas” y sus posibles combinaciones.

- Artículos de los últimos cinco años de publicación.

- Investigaciones que describen su proceso metodológico y cumplen criterios investigativos que garantizan su rigurosidad.

- Investigaciones que a criterio de los investigadores aportan a los intereses de la investigación.

\section{Así también se excluyeron las investigaciones que:}

- No cumplen los criterios de inclusión.

- Investigaciones no relacionadas a la temática.

- Investigaciones que no cumplen con rigurosidad científica de investigación de acuerdo a su proceso metodológico.

- Investigaciones repetidas.

La investigación se realizó desde la búsqueda en motores de investigación científica como:

- Google académico

- Scopus

- Medline

- Researchgate 
- PudMed

- Scielo

- Dialnet

Además, se consideraron como fuentes de investigación válidas, todas aquellas referentes a la bibliografía gris existente, es decir, los repositorios universitarios a nivel nacional e internacional.

Las estrategias de búsqueda utilizadas en la presente investigación fueron mediante palabras clave. Se aplicó inicialmente un primer filtro, relacionado con el año de publicación de los artículos, considerando como los más relevantes aquellos más recientes y desde el año 2017. Posteriormente, en el buscador académico se usaron las palabras clave: "enfermería", "enfermedades crónicas" "área de emergencia” y se las concatenó con los conectores lógicos "and". Estos se describen en el cuadro 1.

Cuadro 1. Estrategias de búsqueda bibliográfica

\begin{tabular}{|l|l|}
\hline \multicolumn{1}{|c|}{ Uso de términos claves } & \multicolumn{1}{c|}{$\begin{array}{c}\text { (paciente crónico) AND (cuidados de } \\
\text { enfermería) AND (emergencia). }\end{array}$} \\
\hline Idioma & Inglés, Español \\
\hline Uso de operadores booleanos & AND, OR, NOT \\
\hline Publicaciones de los últimos 5 años & 2017-2020 \\
\hline Principales fuentes de búsqueda & Google Académico, Scopus. \\
\hline
\end{tabular}

De igual manera, se realizaron búsquedas paralelas con variables modificadas en relación a las originales.

Cuadro 2. Variables originales y modificaciones realizadas

\begin{tabular}{|l|l|}
\hline \multicolumn{1}{|c|}{ Variables originales. } & \multicolumn{1}{|c|}{$\begin{array}{c}\text { Variables con contradicción, implicación o } \\
\text { modificación. }\end{array}$} \\
\hline Emergencia & $\begin{array}{l}\text { Área de emergencia. } \\
\text { Urgencia médica. }\end{array}$ \\
\hline Enfermedades crónicas. & $\begin{array}{l}\text { Pacientes crónicos. } \\
\text { Padecimientos crónicos. }\end{array}$ \\
\hline
\end{tabular}

Mediante estas consideraciones, se procedió a la selección de estudios que se describe:

Selección de los estudios

Los estudios registrados se sometieron a un proceso de selección en forma progresiva que se basó en: 
- Lectura de los títulos de las investigaciones: inicialmente, se dio lectura a las investigaciones, considerando aquellas que son afines a los objetivos de investigación y descartando las que no se aproximan según este criterio.

- Tras la lectura de los títulos y selección del primer filtro de documentos, se procedió a dar lectura a los resúmenes de las investigaciones. En este sentido, se pudo conocer más a profundidad las investigaciones y se descartaron los artículos que estaban repetidos y aquellos que no cumplen los criterios de inclusión, así como los que se enmarquen en los criterios de exclusión.

- Finalmente, tras la lectura de los resúmenes, se procedió a dar lectura completa a los artículos seleccionados, conformando éstos los seleccionados para la investigación.

Gráfico 1. Selección de estudios

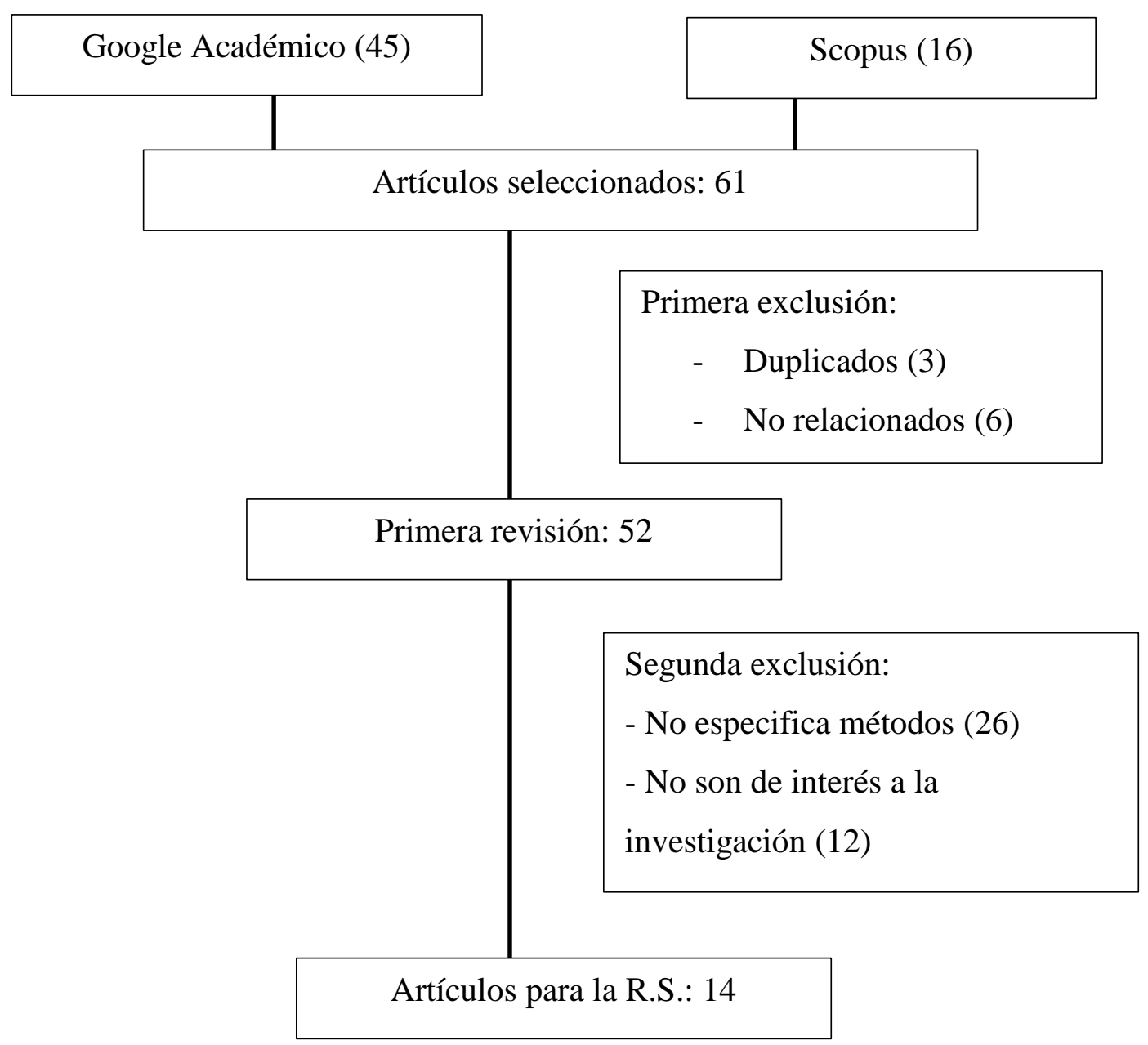




\section{Proceso de extracción de datos}

Los datos fueron extraídos mediante tablas en las que se establecieron los siguientes indicadores para la clasificación de los artículos:

- Año de publicación, autor y país.

- Título de la publicación.

- Objetivos de la investigación

- Métodos de la investigación

- Resultados y conclusiones

- Aspectos relevantes de la investigación

Esta clasificación de los artículos permitió posteriormente agruparlos, compararlos y dar paso a la escritura del artículo de revisión sistémica.

\section{Lista de datos}




\begin{tabular}{|c|c|c|c|c|c|}
\hline $\begin{array}{l}\text { Título del artículo, } \\
\text { autor, año de } \\
\text { publicación }\end{array}$ & Participantes & $\begin{array}{l}\text { Diseño de } \\
\text { estudio }\end{array}$ & Resultados & $\begin{array}{c}\text { Interpretación de los } \\
\text { autores }\end{array}$ & Categorías \\
\hline $\begin{array}{l}\text { Cuidados de enfermería } \\
\text { en pacientes con } \\
\text { insuficiencia respiratoria } \\
\text { tipo I en el servicio de } \\
\text { emergencia adultos del } \\
\text { Hospital Nacional dos de } \\
\text { Mayo. } \\
\text { Gavidia, R. (2017) }\end{array}$ & 2420 pacientes & $\begin{array}{l}\text { Descriptivo } \\
\text { transversal. }\end{array}$ & $\begin{array}{l}\text { El } 38,8 \% \text { de la muestra tuvo o tiene } \\
\text { insuficiencia respiratoria. Este grupo } \\
\text { estuvo conformado por adultos } \\
\text { mayores, en gran parte, que superan los } \\
80 \text { años de edad (5). }\end{array}$ & $\begin{array}{lr}\text { La atención en casos de } \\
\text { emergencia r para } \\
\text { insuficiencia respiratoria } \\
\text { requiere } & \text { tanto } \\
\text { equipamiento médico } \\
\text { como pericia } r \text { y } \\
\text { conocimientos por parte } \\
\text { del personal } & \text { de } \\
\text { enfermería. } & \end{array}$ & $\begin{array}{l}\text { Emergencia. } \\
\text { Insuficiencia } \\
\text { respiratoria. }\end{array}$ \\
\hline $\begin{array}{lr}\text { Cuidado de enfermería } \\
\text { del catéter } & \text { venoso } \\
\text { central en pacientes } \\
\text { renales } & \text { crónicos } \\
\text { sometidos } & \text { a } \\
\text { hemodiálisis. } \\
\text { Zapata, J. (2017) }\end{array}$ & $\begin{array}{l}40 \text { pacientes } \\
\text { crónicos renales. }\end{array}$ & $\begin{array}{l}\text { Descriptivo - } \\
\text { retrospectivo. }\end{array}$ & $\begin{array}{l}\text { Se observa que el hospital no tiene } \\
\text { capacidad operativa para responder a } \\
\text { las exigencias de demanda de la } \\
\text { enfermedad, lo que dificulta el } \\
\text { adecuado procedimiento por parte del } \\
\text { personal de enfermería (6). }\end{array}$ & $\begin{array}{l}\text { Es importante que el } \\
\text { personal de enfermería } \\
\text { trabaje sobre todo en } \\
\text { estrategias de prevención } \\
\text { con enfermedades } \\
\text { crónicas, con objeto de } \\
\text { evitar recurrir a } \\
\text { tratamientos más } \\
\text { complejos que muchas de } \\
\text { las veces no se disponen } \\
\text { en hospitales zonales de } \\
\text { algunas ciudades. }\end{array}$ & $\begin{array}{c}\text { Atención en } \\
\text { enfermería. } \\
\text { Enfermedades } \\
\text { crónicas. }\end{array}$ \\
\hline $\begin{array}{l}\text { Cuidados de enfermería } \\
\text { en pacientes con } \\
\text { traumatismo encéfalo } \\
\text { craneano. } \\
\text { Vilchez, (2019). }\end{array}$ & $\begin{array}{l}37 \text { profesionales } \\
\text { de enfermería. }\end{array}$ & $\begin{array}{l}\text { Descriptivo - de } \\
\text { campo. }\end{array}$ & $\begin{array}{l}\text { El servicio de emergencia no tiene } \\
\text { instrumentos actualizados (manuales de } \\
\text { procedimientos, de organización, } \\
\text { funciones, etc.) y tampoco posee } \\
\text { protocolos y guías que orienten la labor } \\
\text { en cuidados especializados (7). }\end{array}$ & $\begin{array}{l}\text { Las } \\
\text { administrativas dan paso a } \\
\text { que los cuidados de } \\
\text { enfermería en el servicio } \\
\text { de emergencia presenten } \\
\text { falencias. }\end{array}$ & $\begin{array}{l}\text { Atención de } \\
\text { enfermería. } \\
\text { Enfermedades } \\
\text { crónicas. } \\
\text { Emergencia. }\end{array}$ \\
\hline
\end{tabular}




\begin{tabular}{|c|c|c|c|c|c|}
\hline $\begin{array}{l}\text { Manejo de enfermería en } \\
\text { la descompensación del } \\
\text { paciente con diabetes } \\
\text { mellitus tipo } 2 \text { en el área } \\
\text { de emergencia. Fajardo, } \\
\text { G. (2018) }\end{array}$ & 18 pacientes. & $\begin{array}{l}\text { Descriptivo } \\
\text { bibliográfico. }\end{array}$ & $\begin{array}{l}\text { Se identificó que en el área de } \\
\text { emergencia se utiliza el proceso de } \\
\text { atención basado en los patrones } \\
\text { funcionales de Marjory Gordon, el cual } \\
\text { se considera apropiado y eficiente (8). }\end{array}$ & $\begin{array}{l}\text { En el caso de la atención e } \\
\text { pacientes crónicos } \\
\text { (diabetes), se observa que } \\
\text { tras la valoración, } \\
\text { generalmente se requiere } \\
\text { administración de insulina } \\
\text { (en los casos de } \\
\text { descompensación aguda). } \\
\text { Se suma la necesidad de } \\
\text { educar al paciente sobre } \\
\text { los riesgos y consumos } \\
\text { alimenticios. }\end{array}$ & $\begin{array}{l}\text { Paciente crónico } \\
\text { Diabetes } \\
\text { Mellitus. } \\
\text { Cuidados de } \\
\text { enfermería } \\
\text { Emergencia. }\end{array}$ \\
\hline $\begin{array}{l}\text { Cuidados de enfermería } \\
\text { a pacientes con } \\
\text { intoxicación } \quad \text { por } \\
\text { organofosforados en el } \\
\text { servicio de emergencia. } \\
\text { Bautista, G. } 2018\end{array}$ & 39 pacientes. & $\begin{array}{l}\text { Descriptivo } \\
\text { retrospectivo. }\end{array}$ & $\begin{array}{l}\text { En los últimos años se ha mejorado la } \\
\text { información en cuanto al uso de } \\
\text { organofosforados; a pesar de esto, } \\
\text { todavía es frecuente la atención en } \\
\text { servicios de emergencia debido a estas } \\
\text { causas, por lo que se han establecido } \\
\text { protocolos para su atención (9). }\end{array}$ & $\begin{array}{lr}\text { La } & \text { información } \\
\text { oportunidad } & \text { y la } \\
\text { educación } & \text { permiten } \\
\text { mejorar la } & \text { atención por } \\
\text { parte del personal de } \\
\text { enfermería y demás } \\
\text { profesionales } \\
\text { administrativos de los } \\
\text { centros de atención de } \\
\text { salud pública. }\end{array}$ & $\begin{array}{l}\text { Atención de } \\
\text { enfermería } \\
\text { Emergencia }\end{array}$ \\
\hline $\begin{array}{l}\text { Cuidado de enfermería } \\
\text { en adulto mayor con } \\
\text { hipertensión arterial no } \\
\text { controlada, atendido en } \\
\text { el servicio de } \\
\text { emergencia. } \\
\text { Fernández, O. (2018) }\end{array}$ & $\begin{array}{l}\text { Estudio de caso: } \\
1 \text { persona adulta } \\
\text { mayor. }\end{array}$ & Análisis de caso. & $\begin{array}{l}\text { Las personas que asisten al área de } \\
\text { emergencia, en gran parte desconocen } \\
\text { de sus problemas de hipertensión, por lo } \\
\text { que la valoración es importante por } \\
\text { parte de los enfermeros que se basan en } \\
\text { la metodología de dominios y } \\
\text { necesidades afectadas (Virginia } \\
\text { Henderson) y la taxonomía NANDA } \\
\text { (10). }\end{array}$ & $\begin{array}{l}\text { Es preciso diseñar planes } \\
\text { de cuidados validados } \\
\text { mediante enfermería } \\
\text { basada en evidencia. Se } \\
\text { debe extender la atención } \\
\text { a la información y } \\
\text { educación del paciente y el } \\
\text { entorno familiar. }\end{array}$ & $\begin{array}{l}\text { Enfermedad } \\
\text { crónica. } \\
\text { Cuidados de } \\
\text { enfermería } \\
\text { Emergencia. }\end{array}$ \\
\hline
\end{tabular}

Ciencia Latina Revista Científica Multidisciplinar, Ciudad de México, México. 
Proceso de atención de enfermería aplicado a paciente con Insuficiencia Renal Crónica reagudizada e infección de vías urinarias en el servicio de Emergencia.

Flores, M. (2019)

Cuidados de enfermería en un paciente intervenido de hematoma subdural crónico.

Alonso de la Puente, (2017).

Proceso de atención de enfermería aplicado al paciente con cáncer de colon en el servicio de emergencia.

Culqui, R. (2019).

Cuidado de enfermería a un adulto con derrame pericárdico atendido en el servicio de emergencia del hospital docente Las Mercedes. Sandoval, L. (2019)
Estudio de caso:

1 paciente.

Estudio de caso

Mediante la valoración, se establecieron cinco puntos clave a tratar en el paciente ingresado: hipertemia, infección, insuficiencia renal crónica y deterioro de la integridad tisular (11).

Se diseñó un objetivo de cuidado de enfermería basado en las necesidade que surgen tras la intervención

Estudio de caso. 1 paciente.

Estudio de caso.

(funciones afectadas) relacionadas con necesidades musculoesqueléticas, alimenticias y de higiene, enfocando también la prevención complicaciones secundarias (12).

Se identificaron un total de 10 diagnósticos de los cuales se priorizaron 5: dolor crónico, déficit de

Estudio de caso:

1 paciente.

Estudio de caso.

de líquidos, riesgo de infección y disminución de la hemoglobina e inmunosupresión (13)

El plan de atención partió del diagnóstico holístico de las necesidades del paciente. Posteriormente se diseñó

Estudio de caso: 1 paciente.
Estudio de caso un plan de atención basados en la taxonomía NANDA y NIC y Noc (14).
Se logró controlar la hipertemia, se administró medicamento para tratar la infección, se controló parcialmente la insuficiencia renal y el deterioro de la integridad tisular

La enfermería juega un rol fundamental en la prevención de complicaciones; encargándose específicamente no solo de los cuidados, sino también de extender los mismo mediante educación (al paciente y al entorno familiar). Los objetivos orientados a atender los diagnósticos priorizados fueron alcanzados parcialmente, ya que algunos de ellos requieren atención durante tiempos más prolongados con el paciente.

El diagnóstico de parte del personal de enfermería, es fundamental para evitar complicaciones que puedan poner en riesgo la vida del paciente.
Cuidados de enfermería.

Crónico.

Emergencia.

Cuidados de enfermería.

Enfermedad crónica.

Cuidados de enfermería.

Paciente

crónico.

Emergencia.

Cuidados de enfermería.

Paciente

crónico.

Emergencia. 


\begin{tabular}{|c|c|c|c|c|c|}
\hline $\begin{array}{l}\text { Cuidados de enfermería } \\
\text { en el paciente adulto con } \\
\text { crisis asmática atendido } \\
\text { en el servicio de } \\
\text { emergencia. } \\
\text { Valladolid, F. (2017) }\end{array}$ & $\begin{array}{l}10300 \text { pacientes } \\
\text { (historias } \\
\text { clínicas). }\end{array}$ & $\begin{array}{l}\text { Descriptivo - } \\
\text { retrospectivo. }\end{array}$ & $\begin{array}{l}\text { Los datos indican un incremento en el } \\
\text { número de pacientes en los últimos } \\
\text { años (referentes al año de } \\
\text { investigación). Porcentualmente, los } \\
\text { pacientes con crisis asmática son más } \\
\text { mujeres (15). }\end{array}$ & $\begin{array}{l}\text { Se brinda atención oportuna a } \\
\text { los pacientes que muestran } \\
\text { crisis asmática gracias a la } \\
\text { existencia de protocolos } \\
\text { vigentes en la institución. }\end{array}$ & $\begin{array}{l}\text { Emergencia. } \\
\text { Cuidados de } \\
\text { enfermería. }\end{array}$ \\
\hline $\begin{array}{l}\text { Cuidados de enfermería } \\
\text { a pacientes con } \\
\text { hiperglicemia en el } \\
\text { servicio de emergencia. } \\
\text { Castilla, } M .(2018)\end{array}$ & 720 personas. & $\begin{array}{c}\text { Descriptivo - de } \\
\text { campo. }\end{array}$ & $\begin{array}{l}\text { Se determinó mediante el diagnóstico } \\
\text { de los pacientes, que muchos casos de } \\
\text { hiperglicemia corresponden a la ruptura } \\
\text { del punto de quiebre en la inocuidad del } \\
\text { alimento (16). }\end{array}$ & $\begin{array}{l}\text { Se debe enfocar que los } \\
\text { cuidados de enfermería, a más } \\
\text { de brindar asistencia oportuna } \\
\text { en el área de emergencia, } \\
\text { complementan la atención } \\
\text { brindando información } \\
\text { adecuada sobre formas de } \\
\text { prevención de futuros } \\
\text { episodios. }\end{array}$ & $\begin{array}{l}\text { Cuidados de } \\
\text { enfermería. } \\
\text { Pacientes } \\
\text { crónicos } \\
\text { Emergencia. }\end{array}$ \\
\hline $\begin{array}{l}\text { Diagnósticos enfermeros } \\
\text { NANDA más frecuentes } \\
\text { en pacientes con } \\
\text { traumatismo encéfalo } \\
\text { craneano según teoría de } \\
\text { patrones funcionales en } \\
\text { un servicio de } \\
\text { emergencia. } \\
\text { Huamán, F.; Luna, } \\
\text { Pedro; Quillatupa, B. } \\
\text { (2017). }\end{array}$ & 50 pacientes. & $\begin{array}{l}\text { Observacional } \\
\text { de corte } \\
\text { transversal. }\end{array}$ & $\begin{array}{l}\text { Se determinó que el } 78,6 \% \text { de los casos } \\
\text { fueron diseñados en base a la } \\
\text { taxonomía de NANDA. El diagnóstico } \\
\text { más frecuente fue dolor agudo (r/c) } \\
(17) \text {. }\end{array}$ & $\begin{array}{l}\text { De acuerdo al autor, la calidad } \\
\text { de servicios médicos en el área } \\
\text { de emergencia está basada en } \\
\text { dos factores: protocolos y } \\
\text { aptitudes y habilidades del } \\
\text { profesional, que se reflejan en } \\
\text { sus actitudes con el paciente. Si } \\
\text { bien el área de emergencia } \\
\text { supone un área de atención } \\
\text { prioritaria, esto no implica la } \\
\text { desvinculación de factores } \\
\text { éticos y humanísticos en la } \\
\text { práctica. }\end{array}$ & $\begin{array}{l}\text { Cuidados de } \\
\text { enfermería. } \\
\text { Pacientes } \\
\text { crónicos. }\end{array}$ \\
\hline
\end{tabular}




\begin{tabular}{|c|c|c|}
\hline $\begin{array}{l}\text { Prevalencia de } \\
\text { diagnósticos enfermeros } \\
\text { en pacientes crónicos en } \\
\text { emergencia } \\
\text { extrahospitalarias: } \\
\text { mejorando } \\
\text { comprensión de la } \\
\text { complejidad. } \\
\text { Coca, et. Al (2018). }\end{array}$ & $\begin{array}{l}537 \text { pacientes } \\
\text { crónicos. }\end{array}$ & $\begin{array}{c}\text { Descriptivo - } \\
\text { transversal. }\end{array}$ \\
\hline
\end{tabular}

De los pacientes crónicos, el 52,3\%

estuvo conformado por mujeres en

edades medias de 70,8 años, se detectaron: diabetes, cardiopatías, ictus

y enfermedades pulmonar obstructiva

crónica como los principales

antecedentes. El 29,6\% de pacientes

mostró algún grado de dependencia

para concretar las actividades de

cuidado y rutinarias (18).
Los resultados de la

investigación muestran que los enfermeros priorizan en los pacientes crónicos la seguridad, considerando que

frecuentemente se relaciona al grupo poblacional vulnerable de la tercera edad.
Cuidados de enfermería

Pacientes

crónicos.

Emergencia

extrahospitalaria 


\section{RESULTADOS Y DISCUSIÓN}

Se realiza una comparación de los factores que más se destacaron en la investigación, en referencia a la atención de enfermería en el área de emergencia en pacientes crónicos.

La formación y definición de competencias del personal de enfermería destinado al área de emergencias, es cada vez de mayor interés, debido a que, con el incremento de la esperanza de vida, las situaciones que requieren inmediata atención son cada vez más frecuentes. Específicamente, si se centra aún más el foco de investigación en lo que implica las enfermedades crónicas, se tiene un grupo caracterizado en su mayoría por adultos mayores, ya que en estas edades son en las que las personas alcanzan la manifestación crónica de sus enfermedades (19).

Los protocolos de atención de enfermería en el área de emergencia, responden a factores técnicos, así como a habilidades inherentes al profesional, y la convergencia de estos factores son las que permiten brindar la atención oportuna, teniendo en cuenta que el área de enfermería se caracteriza por exigir atención inmediata, en lapsos de tiempo que generalmente se ven limitados.

Por otro lado, y en referencia a las enfermedades crónicas, las mismas se presentan como parte de la atención de emergencia cuando no han sido controladas adecuadamente. Se conoce también que es en el contexto mismo del área de emergencia en el que algunas enfermedades de este tipo son iniciadas para un proceso diagnóstico (10). El desconocimiento de la enfermedad por parte del paciente significa en este sentido un factor de riesgo para afrontar la enfermedad o para prevenir sus complicaciones, por lo que posterior al ingreso al área de emergencia, resulta necesario que se enfoquen factores educativos (9).

En el análisis de documentos, se observa que se enfocan sobre todo grupos de atención prioritaria como son adultos mayores. Algunas investigaciones refieren que la edad media osciló entre los 80 años para los casos de atención de emergencia por insuficiencia respiratoria (5), mientras que en otras se determinó que la edad media fue de 70,8 años (11) (18).

En referencia a los protocolos, se evidencia que la inexistencia de los mismos complica la calidad de atención. Es equivalente a la inexistencia de protocolos cuando éstos no han sido actualizados, ya que de igual manera generan obstáculos sistemáticos que dificultan la atención oportuna (7). En cuanto a quienes sí poseen un proceso de diagnóstico 
oportuno, optan por proceder de acuerdo a la taxonomía NANDA, y NIC NOC (10) (14) (17), siendo citada también los patrones funcionales de Marjory Gordon (8), combinados con las prácticas de cuidados de enfermería basados en la evidencia (10). De acuerdo a este análisis, se evidencia que la existencia de un método garantiza mejores cuidados.

Si bien los métodos garantizan el proceder del personal de medicina, es también cierto que no se está en la capacidad operativa, instrumental, y temporal de atender todas las necesidades manifiestas. En este sentido, se comprende que el primer factor está relacionado con la priorización de los diagnósticos para su atención, generalmente, relacionados con la necesidad de reestablecer la homeostasis en el paciente y tratar el dolor $(13,14,17)$.

La investigación realizada por Coca, et. Al (2020), es un referente claro de las necesidades más frecuentes en los casos de emergencia extrahospitalaria, enfocándose el enfermero en la reducción de riesgos en el entorno, considerando el nivel de dependencia del paciente (18). Otras investigaciones priorizaron el control del dolor, reducción de riesgos de infección, disminución de hemoglobina, (6) (7) (13). En este sentido, es evidente que las prioridades variarán de acuerdo a las características de las enfermedades crónicas, sin embargo, se conoce que estadísticamente gran parte de los casos son atendidos debido a problemas relacionados con diabetes, hipertensión, y cancerígenos (12).

Las conclusiones de las investigaciones orientan a considerar el factor educación, como parte de la acción del enfermero en atención de emergencia, ya que siendo este el primer encuentro con la institución de salud en los casos que se catalogan bajo esta etiqueta, es necesario que desde este encuentro, se orienten los factores de prevención, considerando que gran parte de atención de complicaciones a causa de enfermedades crónicas en el área de emergencia, se deben a factores previsibles (como hábitos alimenticios, por ejemplo), por lo que es fundamental que se eduque en este sentido, con miras a reducir la demanda del área de emergencia que en los servicios de salud pública generalmente opera de forma saturada y sobre su capacidad $(15,16)$.

\section{CONCLUSIÓN O CONSIDERACIONES FINALES}

Se observa que gran parte de las investigaciones citadas responden a estudios de caso llevados a cabo en diferentes áreas hospitalarias. Resultó complejo además la investigación, considerando que generalmente se tratan enfermedades crónicas específicas, siendo las más frecuentes: cáncer, hipertensión, diabetes; por lo que se 
comprende que los diagnósticos en cada tipo de investigación están relacionados específicamente con las complicaciones que derivan de cada una de estas enfermedades. La población que requiere de asistencia de salud en áreas de emergencia por enfermedades crónicas, está caracterizada por adultos mayores (entre 70-80 años). Esto se comprende y se justifica si se tiene en cuenta que muchas enfermedades crónicas presentan complicaciones en edades avanzadas. A su vez, esta característica epidemiológica condiciona y orienta la formación del profesional de enfermería, ya que la adultez mayor posee ciertas características especiales relacionadas con la edad, como grados de dependencia, vulnerabilidad, grupos sociales de poco impacto o apoyo, entre otras.

La existencia de un método de abordaje garantiza un mejor funcionamiento de estas áreas por parte del personal de enfermería; el cual deberá brindar un diagnóstico y atención holística. Se observa que, en el diagnóstico enfermero, se deberá generalmente priorizar los más relevantes para ser atendidos. Algunos de estos casos son remitidos a internamiento, considerando que el restablecimiento de ciertas condiciones de salud, requiere más que la atención puntual de un solo día.

\section{REFERENCIAS BIBLIOGRAFÍCAS}

PAHO. Crecimiento acelerado de la población adulta de 60 años y más edad: reto para la salud pública. [Internet]. 2012. Available from: https://www.paho.org/hq/index.php?option=com_content\&view=article\&id=279 6:2010-crecimiento-acelerado-poblacion-adulta-60-anos-mas-edad-reto-saludpublica\&Itemid=1914\&lang=en.

Menéndez J, Guevara A, Arcia N, León E, Algonso J. Enfermedades crónicas y limitaciones funcional en adultos mayores: estudio comparativo en siete ciudades de América Latina y el Caribe. Rev Pan Salud Pública. 2016;17(5).

Bertoldo P, Ascar G, Campana Y, Martín T, Moretti M. Cumplimiento terapéutico en pacientes con enfermedades crónicas. Rev Cuba Farm. 2016;47(4):468-74.

Serra M. Las enfermedades crónicas no transmisibles y la pandemia por COVID-19. Rev Final. 2020;30(2):1-34.

Gavidia R. Cuidados de enfermería en pacientes con insuficiencia respiratoria tipo I en el servicio de emergencia adultos del hospital nacional 2 de mayo. Callao; 2017.

Zapata J. Cuidados de enfermería del cateter venoso central en pacients renales 
crónicos sometidos a hemodiálisis en el servicio de emergencia . Callao; 2017.

Vílchez P. Cuidados de enfermería en pacientes con trautatismo encéfalo craneano, servicio de emergencia, hospital nacional Hipólito Unánue. Lima; 2019.

Fajardo G. Manejo de enfermería en la descompensación del paciente con diabetes mellitus tipo 2 en el área de emergencia. Machala; 2018.

Bautista G. Cuidados de enfermería a pacientes con intoxicación por organofosforados en el servicio de emergencia del C.S. Nuevo Imperial Cañete 2017. Callao; 2018.

Fernández O. Cuidados de enfermería en adulto mayor con hipertensión arterial no controlada, atendido en el servicio de emergencia. Lima; 2018.

Flores M. Proceso de atención de enfermería aplicado a paciente con Insuficiencia Renal Crónica reagudizada e infección de vías urinarias. Lima; 2019.

Alonso de la Puente S. Cuidados de enfermería en un paciente intervenido de hematoma subdural crónico. Rev Enfr Zamora [Internet]. 2017 [cited 2021 Jan 25]; Available from: https://gredos.usal.es/handle/10366/135834

Culqui R. Proceso de atención de enfermería aplicado al paciente con cáncer de colon en el servicio de emergencia. Lima; 2019 Apr.

Sandoval L. Cuidado de enfermería a un adulto con derrame pericárdico atendido en el servicio de emergencia. Lima; 2019.

Valladolid F. Cuidados de enfermería en el paciente adulto con crisis asmática atendido en el servicio de emergencia del hospital de Apoyo Chulucanas. Callao; 2017.

Castilla M. Cuidados de enfermería a pacientes con hiperglicemia en el servicio de emergencia del C.S. Quimaná. Callao; 2018.

Huamán F, Luna P, Quillatupa B. Diagnósticos enfermeros NANDA más frecuentes en pacientes con traumatismo encéfalo craneano según teoría patrones funcionales en un servicio de emergencia. Lima; 2017.

Coca Boronat E, Díaz Pérez MÁ, Lupiáñez Pérez I, Pérez Ardanaz B, Fuentes Ruíz JÁ, Morales Asencio JM. Prevalencia de diagnósticos enfermeros en pacientes crónicos en emergencias extrahospitalarias: mejorando la comprensión de la complejidad. Emergencias (Sant Vicenç dels Horts) [Internet]. 2020 [cited 2021 Jan 25];211-2. Available from: http://emergencias.portalsemes.org/descargar/prevalencia-de-diagnsticosenfermeros-en-pacientes-crnicos-en-emergencias-extrahospitalarias-mejorando- 
Cuidados de enfermería en pacientes...

la-comprensin-de-la-complejidad/

Gonzáles Ú. Competencias y técnicas de enfermería en los servicios de urgencia. Rev Arch Médico Camaguey. 2018;4(2). 